The cultural space from the revitalization of the heritage of Modernity

PALABRAS CLAVE • ESPACIO CULTURAL · MUSEO $\cdot$ PATRIMONIO MODERNO - HIBRIDACIÓN - RECONVERSIÓN

KEYWORDS - CULTURAL SPACE . MUSEUM . MODERN HERITACE - HYBRIDIZATION - REVITALIZATION

\section{RESUMEN}

El espacio de la cultura y el arte se ha convertido en arquetipo privilegiado de la actividad global de nuestras sociedades en los países industrializados, en espacio de confluencia entre fuerzas políticas $y$ sociales, económicas y tecnológicas, o estratégicas y mediáticas. En el ámbito europeo, en las últimas décadas, ha destacado la tendencia por reconvertir el patrimonio industrial de numerosas ciudades mediante espacios destinados precisamente a la cultura, emergiendo nuevas situaciones en las que late el estado anterior al cambio, herencia de la modernidad. El artículo clasifica y analiza una serie de estrategias mediante proyectos paradigmáticos que responden al actual estado de la cultura en la sociedad global, así como a la oposición entre el ideal moderno de "especificidad" y el contemporáneo de "hibridación". Desde la lógica de hibridación, estos espacios culturales también abren nuevos horizontes para repensar una relación más activa entre arquitectura, arte y observador.

\section{ABSTRACT}

The space of culture and art has become the privileged archetype of the global activity of our societies in industrialized countries, in confluence space between politicat and sociat, economic and technological, or strategic and media forces. At the European level, in recent decades can stand out the trend to revitalize the industrial heritage of many cities through spaces destined precisely to culture, emereing new situations in which emerges the sta before the change, the heritage of modernity. The article classifies and analyses through paradigmatic projects several strategies that respond to the current state of culture in our global society, as well as the opposition between the modern ideal of "specificity" and the contemporary of "hybridization". From the logic of hybridization, these cultural spaces also open new perspectives to rethink a more active relationship between architecture, art, and the observer.

\title{
El espacio cultural desde la reconversión del patrimonio de la Modernidad
}

\author{
DANIEL BARBA RODRÍGUEZ · Universidad de Valladolid · barba.rodriguez.daniel@gmail.com \\ JAVIER DE ESTEBAN GARBAYO · Universidad de Zaragoza· jdegarbayo@gmail.com \\ Fecha de recepción: 30 de marzo de 2021 · Fecha de aceptación: 29 de mayo de 2021
}

\section{INTRODUCCIÓN}

El espacio del museo y la cultura se ha convertido en las últimas décadas en arquetipo privilegiado de la actividad global de nuestras sociedades en los países industrializados, en espacio de confluencia de fuerzas políticas y sociales, económicas y tecnológicas, o estratégicas y mediáticas. Tal como apunta Bauman, en esta época de totalidades supralocales, de "comunidades imaginadas" fruto de la movilidad y el intercambio de la modernidad, estos espacios se convierten en lugares para la construcción de narraciones e identidades culturales fabricadas, postuladas o edificadas (Bauman, 2011). Como expresión de la cultura y el arte, juegan un papel esencial en la construcción de una estructura social, una naturaleza de lo común, que consiste en una red de interdependencias desarrolladas a través de la interacción humana.

Desde el cuestionamiento de la idea de museo tradicional, algunos de los espacios culturales más recientes han planteado un interesante debate en torno a la producción, difusión y recepción del arte contemporáneo. Uno de estos temas es la vigencia de la idea de narración que ha predominado la concepción del museo a lo largo del siglo XIX y gran parte del siglo XX. Se trata de alejar al museo de fomentar un gran relato de una historia que nos narra acerca de nosotros mismos, de la materialización de un texto histórico que insufla a éstos un aura y un espíritu épico. También de convertirse en mera compilación, en almacén para botines de guerras culturales, casa de tesoros, sitio donde amontonar objetos de valor para la burguesía o el turista. Se trataría, como señala Sloterdijk, de implicar a la sociedad actual en una comunicación inteligente con lo extraño y lo propio, de acometer la descolonización interna de la cultura'?

No menos interesante resulta la reflexión acerca de los criterios de intervención en estos lugares. En las diversas estrategias que analizaremos, veremos cómo el patrimonio arquitectónico heredado es reconvertido en espacio expositivo, emergiendo una tensión entre la historia (el valor documental del edificio) y la contemporaneidad (su reciclaje desde una nueva función y oportunidad). El sociólogo Ulrich Beck ha argumentado al respecto, que la modernidad, en su segunda fase en la que vivimos, se ha convertido en reflexiva, preocupada por modernizar su vieja infraestructura o en reinstrumentalizarla. En este sentido, los proyectos que veremos a continuación plantean la

Señala Slotedijk que cualesquiera sean los aspectos del mundo del pasado y del presente que estos museos puedan exhibir, sólo encontrarán su momento histórico si introducen a su público en un extrañamiento ilustrado del mundo (Sloterdijk, 2015). 
1. (Izquierda) Palais de Tokyo, París, 2012-14. (Derecha) FRAC, Dunquerque, 2013. Anne Lacaton \& Jean-Philippe Vassal. Fuente: www.lacatonvassal.com / Fotógrafo: Philippe Ruault.
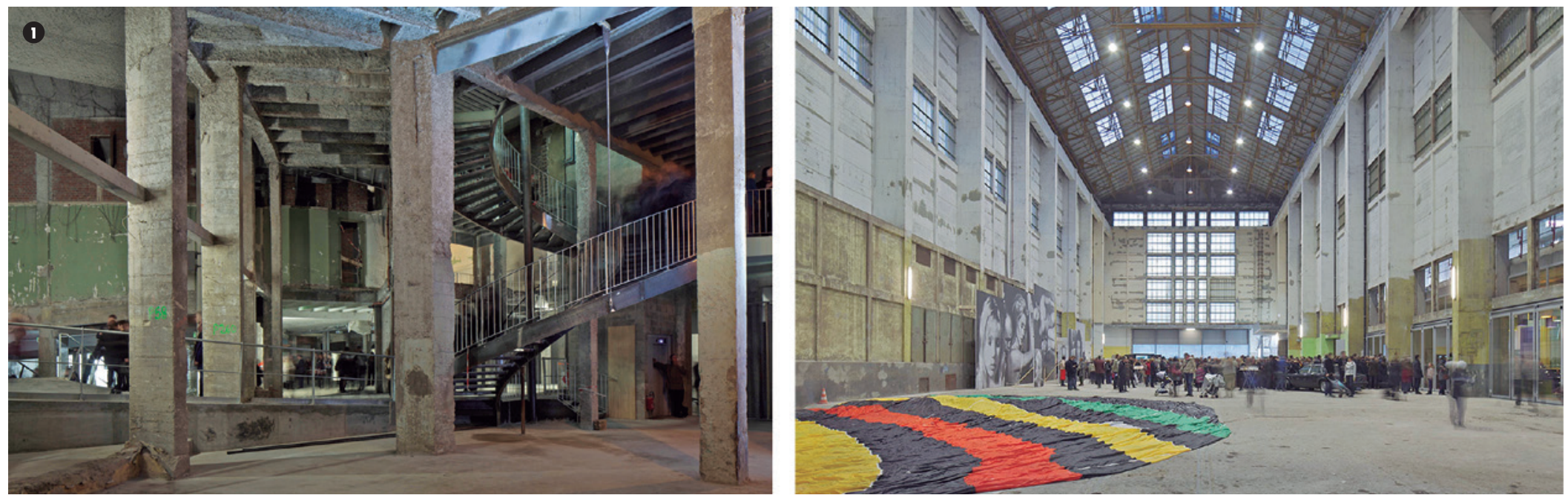

reconversión de antiguos espacios de la modernidad en renovados centros culturales como respuesta a la actual economía postindustrial (Beck, 1998). La oposición entre el ideal moderno de "especificidad" y la estrategia contemporánea de "hibridación" emerge en los distintos casos, en los que la construcción de nuevas identidades compartidas como reflejo de la cultura global se acompaña de la regeneración de un entorno que se afirma como espacio de lo común. Desde esta lógica de hibridación, estos espacios culturales también abren nuevos horizontes para repensar una relación más activa entre arquitectura, arte y observador.

\section{EL ESPACIO DE LA CULTURA COMO "INSTALACIÓN"}

Una de las estrategias destacadas de reconversión del patrimonio heredado de la modernidad ha sido la intervención en su naturaleza espacial como si de una "instalación" se tratara. La idea de "instalación" plantea un equilibrio entre la reconsideración del espacio expositivo como lugar activo que fomenta la experiencia artística donde los observadores se mueven entre las distintas obras en un lugar que altera las jerarquías del museo tradicional, y la de un espacio de representación. Esta estrategia, por lo general, plantea la manipulación del edificio heredado, pudiendo hablar sin duda de una nueva espacialidad, con el fin de facilitar la experimentación y la producción artística ligada a la propia naturaleza del espacio expositivo. Frente al museo tradicional que impone una distancia con las obras, esta estrategia fomenta una estética espacial más abierta, en muchos casos mediante un estado de lo "no-acabado", que trata de favorecer el intercambio entre obra y observador desde el diálogo entre la naturaleza representativa de la obra y su sentido de presencia (Mitchell, 2015).

Uno de los casos paradigmático en la cultura artística europea más reciente que ejemplifica esta estrategia es el Palais de Tokyo de París. Este centro cultural recoge las ideas de sus primeros directores, Nicolas Bourriaud y Jerome Sans, quienes, frente al concepto tradicional de museo, conciben el Palais como un lugar de producción, donde la obra de arte no actúe como mero objeto de contemplación, sino más bien como agente activo, como relato que continúa y reinterpreta relatos anteriores desde la generación de comportamientos y potenciales reutilizaciones (Hernández Martínez, 2013). Como señala Nicolas Bourriaud en su texto Estética relacional: "la obra de arte puede consistir en un dispositivo formal que genere relaciones entre personas o surgir de un proceso social, un fenómeno que he descrito con el nombre de estética relacional cuya principal característica es considerar el intercambio interhumano en tanto que objeto estético de pleno derecho" (Bourriaud, 2008, p. 35).

La propuesta de los arquitectos Lacaton y Vassal, ganadores del concurso, trata de favorecer esta concepción desde un diálogo crítico con el edificio anterior y lograr una "naturaleza espacial abierta" que beneficie la diversidad expositiva (Badía, 2012). La intervención opera en términos artísticos convirtiendo el edificio existente en object trouvé (objeto encontrado), un ready-made donde la naturaleza previa es alterada adquiriendo un nuevo significado. La propuesta o "instalación", como los mismos autores denominan, supone una re-instrumentalización de la arquitectura previa, favoreciendo un renovado espacio con ciertas trazas dadaístas. Si bien la propuesta supone una puesta en valor de lo existente, aunque alterando la lógica espacial previa, también denota la propia arbitrariedad de lo "encontrado", donde los elementos más singulares adquieren un nuevo valor como se aprecia en las plantas inferiores, donde se afirma su naturaleza plástica. Se proyecta con un gusto por lo artístico, lo contingente, lo poético, aplicando, a su vez, una concepción específica a los términos "memoria", "historia" o "patrimonio" (Rorty, 1989) (FIgURA 1). 
2. Palais de Tokyo, París, 2012-14. Anne Lacaton \& Jean-Philippe Vassal. Fuente: Dibujo de elaboración propia, 2021.
3. FRAC, Dunquerque, 2013. Anne Lacaton \& Jean-Philippe Vassal. Fuente: Dibujo de elaboración propia, 2021.

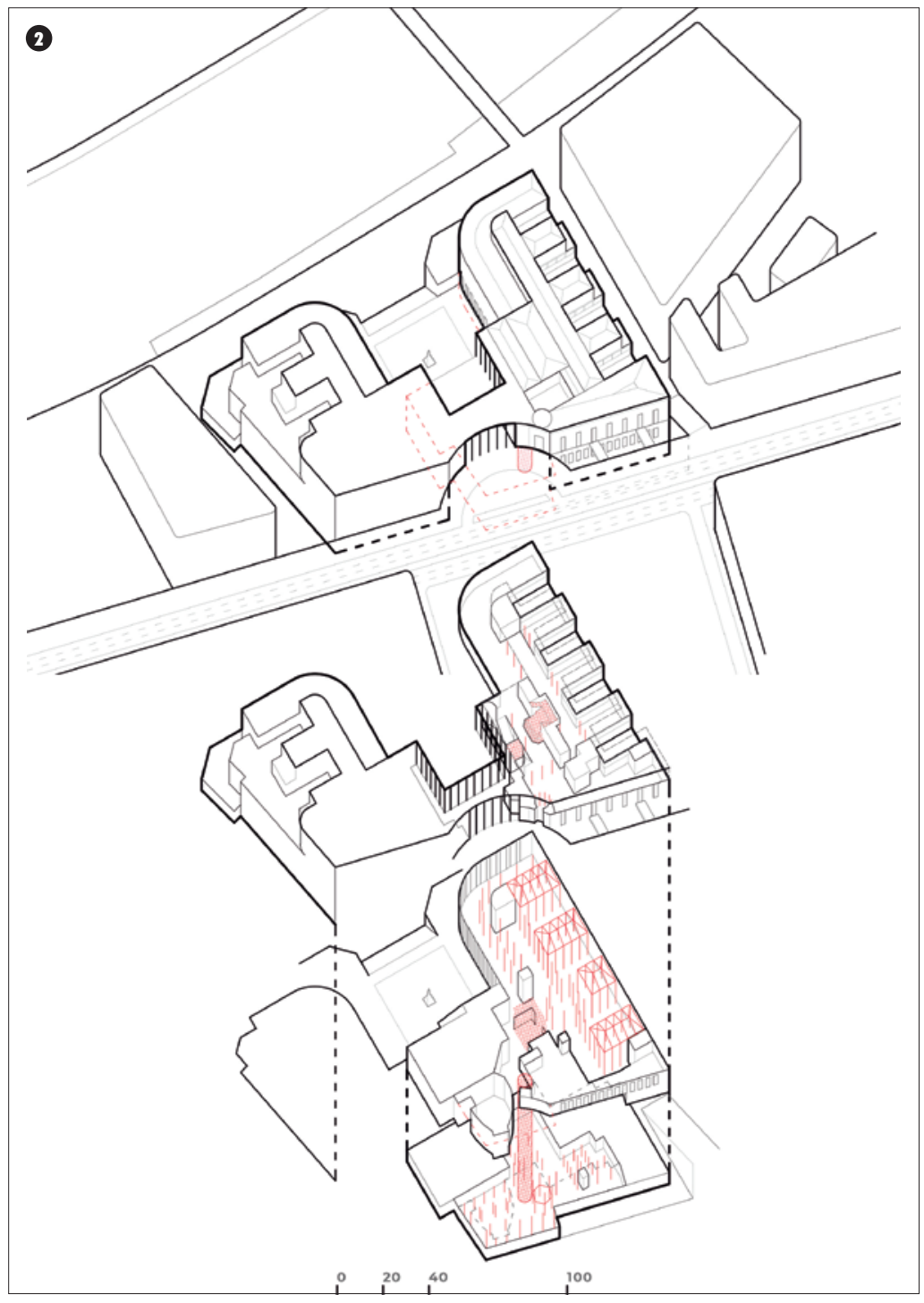

EI FRAC de Dunquerque resulta otro buen ejemplo de cómo el espacio de la cultura responde a esta idea de "instalación". La antigua nave AP2 también se convierte en object trouvé, objeto singular y emblemático dispuesto para enriquecer la actividad del nuevo barrio. Su espacialidad viene acentuada por su vaciamiento, para ofrecer un potencial de uso en relación con otros espacios contemporáneos de la cultura como la sala de turbinas de la Tate Modern de Londres. La individualidad de este volumen también se reconvierte mediante la disposición de uno contiguo, "sombra transparente del existente", que es atravesado por una pasarela pública que conecta exterior e interior. Si bien la nave previa se expresa como espacio disponible, un "espacio encontrado" a reinterpretar, su existencia es posible gracias a la aparición de la nueva nave que almacena los usos necesarios, acentuando un diálogo mutuo de interdependencia e individualidad, como expresión, o quizá metáfora, de la condición existencial en el mundo global actual (Lacaton y Vassal, 2011).

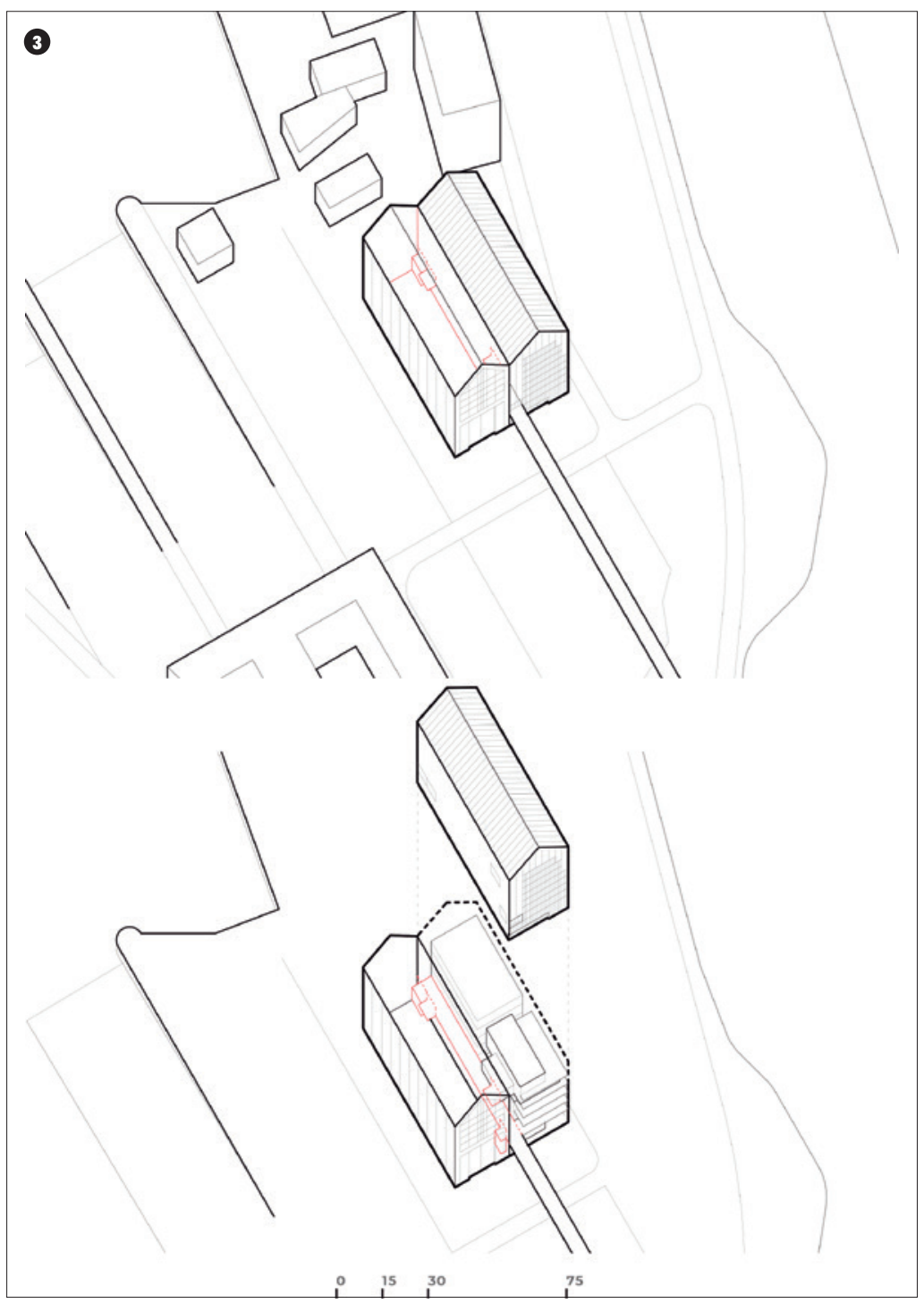

El FRAC, del mismo modo que el Palais de Tokyo, supone una reflexión acerca de la condición del arte y el espacio de la cultura, entendido como un lugar público permeable, inestable, abierto al cambio, a las experiencias y a los encuentros entre productores (artistas o comisarios) y público, un lugar de producción y no solo de exhibición. El continuo desplazamiento de la naturaleza de la obra de arte iniciado con las vanguardias de comienzos del siglo veinte, da un nuevo paso, ya que el encuentro crítico, activo entre creadores, 
comisarios y público, pasa a formar parte de la misma obra. Esta hibridación entre todos los agentes, reflejo de la idea de "instalación", es lo que configura la experiencia artística y donde el espacio cobra un papel activo para facilitar una ocupación más creativa (FIgURAs 2 y 3)².

\section{EL ESPACIO DE LA CULTURA DESDE LA LÓGICA DE LA MODA}

Un tema ineludible en el contexto global actual a la hora de reflexionar sobre el espacio de la cultura es su relación con la moda, más si cabe en un momento donde ésta ha asumido el protagonismo en la iniciativa de establecer el cambio constante como norma de la vida humana. Observamos que destacadas firmas a nivel mundial se han convertido en promotoras de algunos de los centros dedicados al arte y la cultura de mayor proyección. Como señala el filósofo Georg Simmel: "La moda nunca se limita a ser. Existe en un permanente estado de devenir"; una naturaleza vital que no pierde su ímpetu y que se transforma en una fuerza impulsora que no cesa de acumularse. Este "devenir" asume los deseos contradictorios ligados al afán por obtener un sentido de pertenencia en el seno de un grupo, aparejado a una búsqueda de ser como los demás, y al mismo tiempo original; un conflicto donde la moda parece asegurar el compromiso entre la tendencia a la igualdad social y a la individualidad ${ }^{3}$.

Al mismo tiempo, el estilo de vida declarado por quienes ya lo tienen o aspiran a él, que se comunica a los demás mediante las modas cambiantes, se define tanto por la prominente exhibición de los signos de las últimas tendencias como por la ausencia de los que ya no están a la moda. Una vez que los esfuerzos concertados

4. Fondazione Prada, Milan, 2008-18. Rem Koolhaas/OMA. Fuente: Arquitectura Viva / Fotógrafo: Bas Princen.

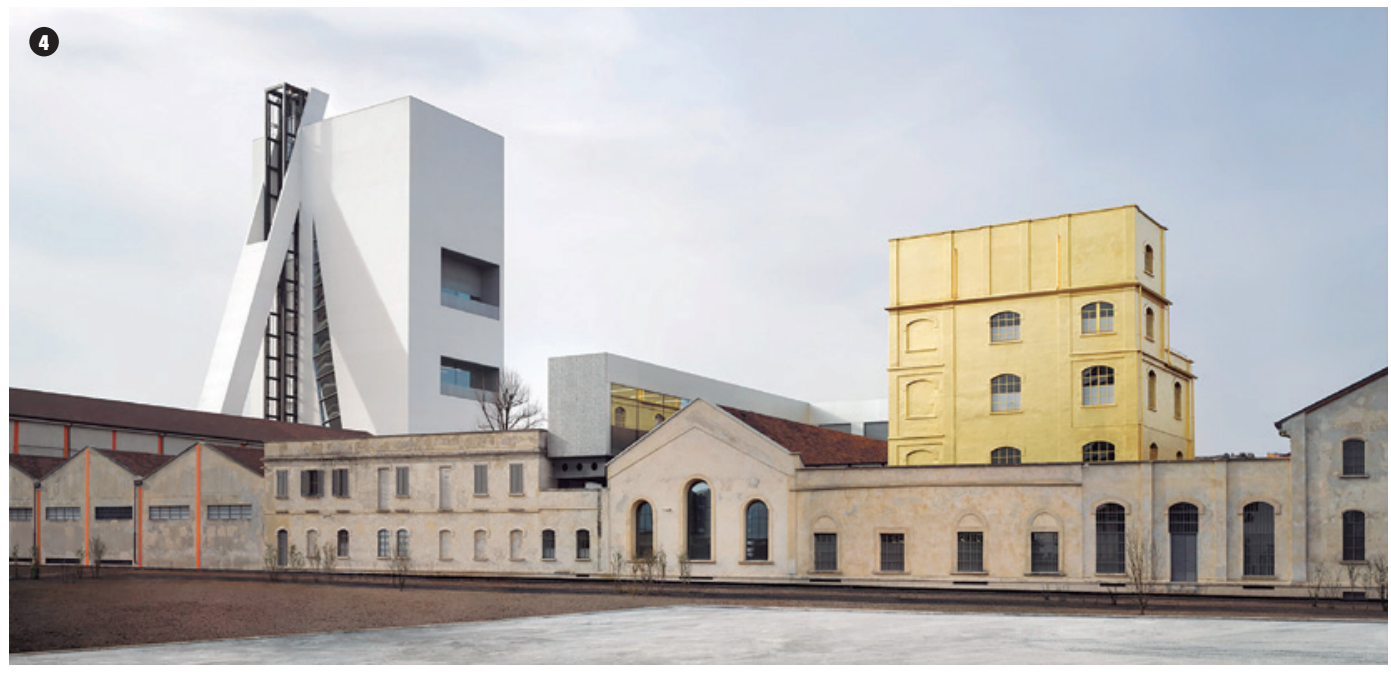

e ingeniosos del mercado de consumo han permitido que la cultura se deje subyugar por la lógica de la moda, se vuelve necesario (para ser uno mismo y ser visto como tal) demostrar la capacidad de ser otra persona. El modelo personal en la búsqueda de la identidad pasa a ser el del camaleón, ya que la cultura exige que adquiramos la destreza de cambiar nuestra identidad (o al menos su manifestación pública) con tanta frecuencia, velocidad y eficacia como cambiamos de camisa o de ropa interior.

Desde este punto de vista, Fondazione Prada (Rem Koolhaas/OMA) resulta uno de los casos paradigmáticos para valorar la relación entre cultura y moda, más aún si atendemos a su emergente papel en la ciudad de Milán, urbe identificada desde hace décadas por su destacado papel en el ámbito de la moda internacional ${ }^{4}$. La propuesta se mueve precisamente en la contradicción de un lugar que aspira a una renovada imagen sin perder por ello la identidad del espacio industrial previo (una antigua destilería de ginebra). Como señalan sus autores: "La Fondazione no es un proyecto de preservación ni una nueva arquitectura. Ambas condiciones, habitualmente separadas, se confrontan una a la otra en una permanente interacción, ofreciendo un ensamblaje de fragmentos que no fijan una única imagen". Lo nuevo, viejo, horizontal, vertical, amplio, estrecho, blanco, negro, abierto o cerrado, establecen una hibridación espacial que revela la complejidad y el "inestable programa" de la propuesta (Abrahams, 2015). Fondazione Prada parece escenificar la relación (o confusión) entre consumidor y observador, ligado a lo que la moda le ofrece, confusión que da lugar a una estrategia sin temor a que se juzgue sobreactuada. La definición e introducción de los camaleónicos cuerpos (un pabellón de exposiciones, una torre y un cine) revela la noción de artificio que parece no disimular Rem Koolhaas/OMA, como se evidencia en el lustro de cada una de las fachadas (principalmente en el uso del dorado),

2 Señala Hal Foter que "las obras para un sitio específico pueden utilizarse para hacer que estos no-espacios parezcan específicos de nuevo, para reajustarlos como lugares concretos, no espacios abstractos, en términos históricos y/o culturales [...] valores como la autenticidad, la originalidad y la singularidad pueden retornar como propiedades de sitios que a los artistas se les pide que definan o embellezcan" (Foster, 1996, p. 201).

3 Esta solución de compromiso no puede prolongarse en un estado permanente, no puede establecerse de una vez y para siempre, la cláusula "hasta nuevo aviso" está inscripta en ella con tinta indeleble y exige renegociación continua (Bauman, 2013).

4 Otros proyectos como Pirelli Hangar Biccoca (2012) o Gucci Hub (2016) también pretenden actuar como instrumento para revitalizar la actividad cultural de la ciudad al tiempo que renovar su identidad urbana. 
para una actitud entre el atrevimiento y la falta de prejuicios. Todo ello persigue una condición atmosférica en la que observadores, consumidores y obras interaccionan en un permanente intercambio (Hays, 2016) (FIgURA 4).

Fondazione Prada, a su vez, permite entender cuánto la arquitectura de Koolhaas ha adquirido como condición sine qua non la puesta en cuestión de los principios arquitectónicos modernos. La definición de cada uno de los diversos cuerpos se aleja de la búsqueda de una identidad inequívoca, aspecto celebrado por el propio Koolhaas como un hallazgo tipológico, sin que deje de latir por ello la naturaleza industrial previa (Masoero, 2018). La propuesta también acentúa una tensión constante entre lo genérico y lo singular, en referencia al propio "devenir" de la moda si nos remitimos a la interpretación ofrecida por Georg Simmel.

Desde esta noción, lo genérico para Koolhaas se libera del corsé de una identidad inmutable para alterar el ciclo de independencia que supone el límite de la identidad (Koolhaas y Foster, 2013). En cuanto a esta tensión entre lo genérico y lo singular, la Fondazione remite por momentos a algunas pinturas metafísicas del artista italiano Giorgio de Chirico, pensemos en sus oníricos paisajes urbanos ${ }^{5}$. Esta visión también se aprecia en la yuxtaposición y el tratamiento (lustro) de los camaleónicos cuerpos. En este sentido, cabe destacar, las trazas surrealistas de la intervención, movimiento que sedujo a Koolhaas desde sus inicios, y que en este sentido sirve para cuestionar la idea moderna de la racionalización de la apariencia física de la obra arquitectónica.
5 Cabe señalar la influencia de la pintura metafísica de Chirico en la arquitectura de Aldo Rossi, principalmente sus visiones urbanas. En ambos destaca la dialéctica entre los volúmenes que componen la escena urbana y el espacio público de corte metafísico. Así, la influencia de Chirico en la concepción visual de la Fondazione también puede entenderse como un guiño a Aldo Rossi por parte de Rem Koolhaas precisamente en su ciudad natal.
5. Fondazione Prada, Milan, 2008-18. Rem Koolhaas/OMA. Fuente: Dibujo de elaboración propia, 2021.

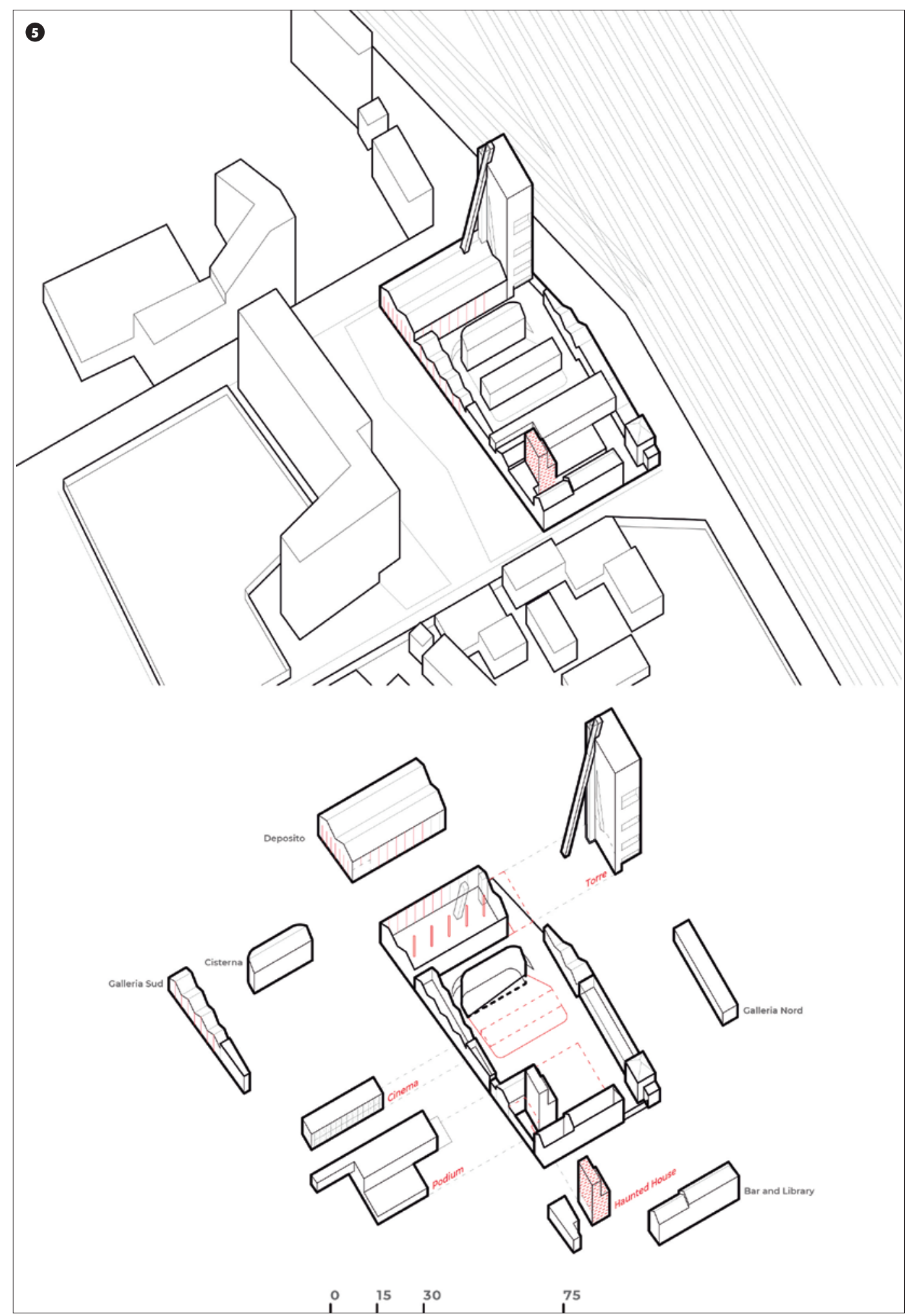


Junto a ello, la propuesta plantea una reflexión acerca de la contingencia entre las necesidades y los medios presentes, para una transformación de lo real en lo posible. Se pretende con ello dar vía libre a lo posible, sin presupuestos que definan cuáles son los atributos últimos que debe tener lo construido, ya que en última instancia este hecho no agota lo que será su uso (Moneo, 2019). Desde la aspiración por lo posible, Koolhaas no trata de hallar una identidad (un "ser"), sino un marco de experiencias y contenidos (un "devenir"). Las "situaciones" emergentes que caracterizan la Fondazione, la hibridación entre lo nuevo y lo viejo, lo cotidiano y lo singular, o incluso lo metafísico y la realidad física, revelan que el modo de estar y vivir un edificio, su existencia, en este caso ligado a lo efímero de la moda como ingrediente esencial de la cultura contemporánea, solo puede construirse desde una identidad siempre abierta al cambio (FIgUra 5).

\section{EL ESPACIO DE LA CULTURA DESDE LA "ARQUITECTURA BODEGONISTA"}

En la reconversión de edificios industriales en espacios para la cultura y el arte, algunas propuestas recientes han abierto nuevas ópticas en la comprensión de su naturaleza visual. No se trata únicamente de restaurar su valor patrimonial, sino más bien, de interrogarlo con el fin de hallar pautas para operar sobre la realidad. La naturaleza interna de la organización material puede brotar así en una pregnancia que nos permite cuestionar las estructuras establecidas de significación. No se trata de detenerse en la descripción de fenómenos sino hacerlos legibles, de disolver las formas conocidas de la experiencia y su fuerza estructurante para recuperar la libertad (o buscar nuevas formas) de conceptualizar y percibir la materia. Quizá encontremos, de este modo, un lugar intermedio entre la precisión y la estructura de quien encuentra universos de diferencia entre ligeras deformaciones del trazo y el gesto (arquitectura del retrato) y la riqueza de textura y sensualidad de aquella que es capaz de abandonarse a la realidad de la materia (arquitectura del paisaje). ¿̇Podría hablarse en ciertos casos de "arquitectura bodegonista"?

Esta aproximación requeriría, en principio, la ampliación de la noción del bodegón desde el cuestionamiento de lo real. Quizás así pueda relacionarse la obra con una mirada de estructura frágil, eliminando parte de la carga simbólica, pero evocando a la vez una profundidad más allá de la superficie. El filósofo italiano Gianni Vattimo argumenta a favor de una arquitectura de "imagen frágil" en "El fin de la modernidad", más interesada en la interacción asociativa y sensorial que en la construcción simbólica de un ideal. Esta aproximación también plantea un dialogo entre exterioridad e interioridad desde una condición más intima y afectiva de lo visual, añadiendo textura a nuestra compresión de lo icónico en la arquitectura. Si el animal es atraído en relación con la superficie, el ser humano lo es en relación con lo que hay detrás, con el fin de responder a la cuestión sobre lo real y entablar un diálogo. Algo de esto sucede en los bodegones de Giorgio Morandi, donde se produce un cuestionamiento de lo real pese a que el autor extrae su iconografía de la realidad más cotidiana. Así, si algunos modernos buscaron transcender la figura referencial y algunos posmodernos deleitarse en la imagen, lo que podríamos perseguir ahora sería poseer la cosa real, aún conscientes de la dificultad o imposibilidad de este empeño (Foster, 2010).

Una de las propuestas que nos permite avanzar en esta aproximación es la ampliación del Museo Küppersmühle realizada por Herzog $\&$ de Meuron en Duisburgo, basado en la reconversión de un edificio industrial de ladrillo de principios del siglo XX, emblema de la regeneración de esta zona portuaria de la ciudad. La presencia del edificio existente (un aglomerado de prismas de ladrillo y silos de chapa roblonada) se acentúa mediante el tapiado parcial de las ventanas originales y por las nuevas incisiones (ventanas) de formato vertical (en relación con la dimensión de las salas interiores). Esta operación supone un cuestionamiento del orden visual anterior a través de la manipulación de la escala de conjunto. La fachada al río adquiere así el ritmo abstracto de una partitura que redescubre un orden interno alternativo, sin abandonar del todo el anterior, acentuado por la iluminación desde el interior cuando anochece. En la nueva ampliación que los arquitectos suizos plantean en 2013, se suman unos nuevos cuerpos cerámicos que refuerza la estrategia bodegonista previa y la interpretación visual del conjunto. Es la suma de unidades lo que cuenta, lo que configura un marco interpretativo que nos permite observar lo anterior, la realidad existente transformada por la nueva operación, con otros ojos. Se manifiesta con ello la naturaleza híbrida de un conjunto que oscila entre "la tradición, el Art Deco y la hipermodernidad" (Herzog y de Meuron, 2018) (FIGURA 6).

Otra intervención en sintonía con la aproximación de Herzog \& de Meuron en Duisburgo, es la llevada a cabo por Caruso St John en la Newport Street Gallery de Londres. La propuesta se mueve a medio camino entre la irreverencia y el respeto por lo existente para lograr una solución que expresa una poética yuxtaposición de lo nuevo con lo viejo. La articulación de los distintos cuerpos cerámicos afirma el diálogo que resignifica el conjunto, su naturaleza visual, pero que no niega la condición individual de cada uno de ellos (Woodman, 2016). La imagen que se extrae de este diálogo deviene así en campo de experimentación, por encima de la organización espacial o estructural. La envolvente se convierte en superficie de articulación entre interior y exterior, lugar donde se registran los valores públicos del objeto arquitectónico. Por otro lado, la superposición de los distintos cuerpos adquiere aquí un sentido muy diferente al del object type de la producción industrial. Se trata de elaborar un material cada vez más rico y consistente, capaz de captar diferencias cada vez más intensas. La naturaleza bodegonista del conjunto también expresa una relación conceptual entre cada unidad volumétrica, ligada tanto a la estructura sintáctica del objeto arquitectónico como a su relación con el contexto. 
6. (Izquierda) Ampliación del Museo Küppersmühle, MKM, Duisbugo, 2013-. Herzog \& de Meuron. (Derecha) Newport Street Gallery, London, 2015. Caruso St John. Fuente: www. herzogdemeuron.com_www.carusostjohn.com / Fotógrafa: Hélène Binet.
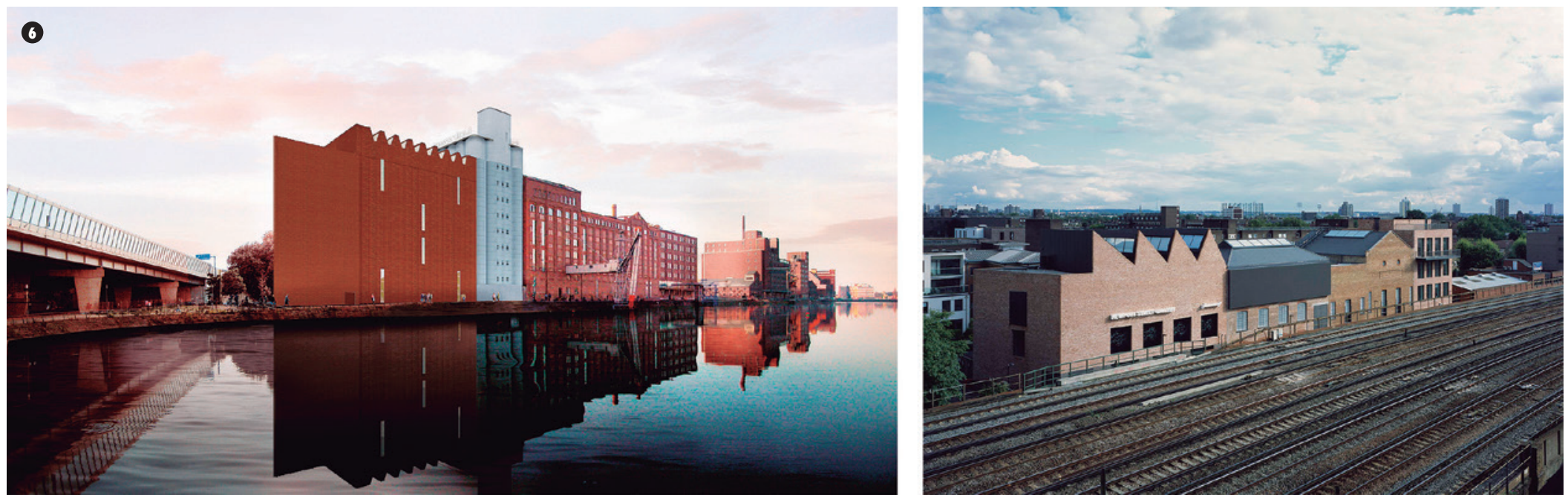

En ambas obras el modo en cómo se articulan los distintos cuerpos que conforman las volumetrías, objetos en apariencia cotidianos, también muestra un interés por el arte pop, interpretación que no dista de la que podemos encontrar en artista como Jasper Johns, Claes Oldemburg o Richard Hamilton; una reflexión sobre la visualización de estos objetos, su realidad material palpable, o acerca de una naturaleza visual cuando no están sometidos a la economía del espectáculo. En este caso, la combinación de imagen y cosa se relaciona con el "objeto encontrado", tratando de vencer la dicotomía entre imágenes y cosas, la escisión de la realidad y la alienación de los objetos (Figuras 7 y 8 ) $^{6}$.

El Pop Art de los sesenta y setenta planteó una novedosa aproximación al bodegón. Es el caso de las latas de sopa Campbell de Andy Warhol, donde la imagen modificada del producto comercial sustituye al objeto físico, o la obra de Roy Lichtenstein "Bodegón con una pecera de peces de colores" (1972), que combina los colores puros de Matisse con la iconografía pop de Warhol. Dentro del neodadaísmo, Jasper Johns volvió a la representación tridimensional de Duchamp para crear su propio tipo de bodegón, como en "Bronce pintado" (1960) o "Casa de locos" (1962). El auge del fotorrealismo ha reafirmado la representación ilusionista, al tiempo que una fusión de objeto, imagen y producto comercial (Don Eddy y Ralph Goings). Las obras digitales recientes también han expandido las fronteras del bodegón.

\section{EL ESPACIO DE LA CULTURA COMO "INFRAESTRUCTURA URBANA"}

Otra estrategia a destacar en cuanto a la reconversión del patrimonio de la modernidad es aquella que asume la naturaleza física de lo heredado para concebirla como infraestructura, esto es, como soporte para la organización funcional y estructural del evento cultural que albergará en su interior. No se trata de transformar la identidad previa, sino de operar desde su lógica estructural o su imagen urbana. En muchos casos, se une a ello, una privilegiada situación que favorece su relevancia como agente urbano. Un ejemplo paradigmático de esta concepción es KANAL-Centre Pompidou, que nace de la transformación de una fábrica de automóviles de la firma Citroën situada en un área céntrica de Bruselas. La propuesta asume la identidad de la factoría previa y desde una economía de medios convierte el espacio en una renovada factoría, en lugar para la creatividad y la producción cultural. Además, como sucedió con el primer Pompidou de París definido como "catalizador para la regeneración urbana" y que supuso la gentrificación gradual del Marais, KANAL (emblema del plan director de la zona) trae consigo la vocación de revitalización, a modo de infraestructura urbana para el intercambio cultural entre la población local y el esperado turismo.
Una de las cualidades urbanas más destacadas de KANAL, por lo tanto, es el mantenimiento de la identidad y el aura fabril del edificio previo, testimonio de la primera modernidad. Se conserva la noción de solar denso a nivel psicológico, donde la historia y la memoria se convierten en cualidades materiales palpables, sin impedir por ello el desarrollo de nuevas experiencias artísticas y encuentros sociales. El eslogan de la propuesta, "un optimismo radical que confía en lo existente", indica cierta distancia respecto al gesto espectacular tan frecuente en este tipo de espacios, para enfatizar una actitud "crítica y receptiva". Asumiendo la complejidad urbana que la regeneración de toda la zona conlleva, el nuevo espacio aspira a convertirse en motor e infraestructura del cambio. A este término también se asocian otros como "atmósfera", "energía" o "experiencia" (empleados por sus autores), ligando la propuesta al camino recorrido por el arte durante las últimas décadas. La propuesta, con ello, atiende a determinados deslizamientos que se han producido en el arte con la llegada del siglo XXI, como las nuevas intervenciones sobre el espacio, las diferentes construcciones de la visión y las definiciones ampliadas del arte.

KANAL también supone una aportación más a la crítica del museo suscitada en el contexto actual y que trata de desmontar el aura con la 
7. Ampliación del Museo Küppersmühle, MKM, Duisbugo, 2013-. Herzog \& de Meuron. Fuente: Dibujo de elaboración propia, 2021.
8. Newport Street Gallery, Londres, 2015. Caruso St John. Fuente: Dibujo de elaboración propia, 2021.
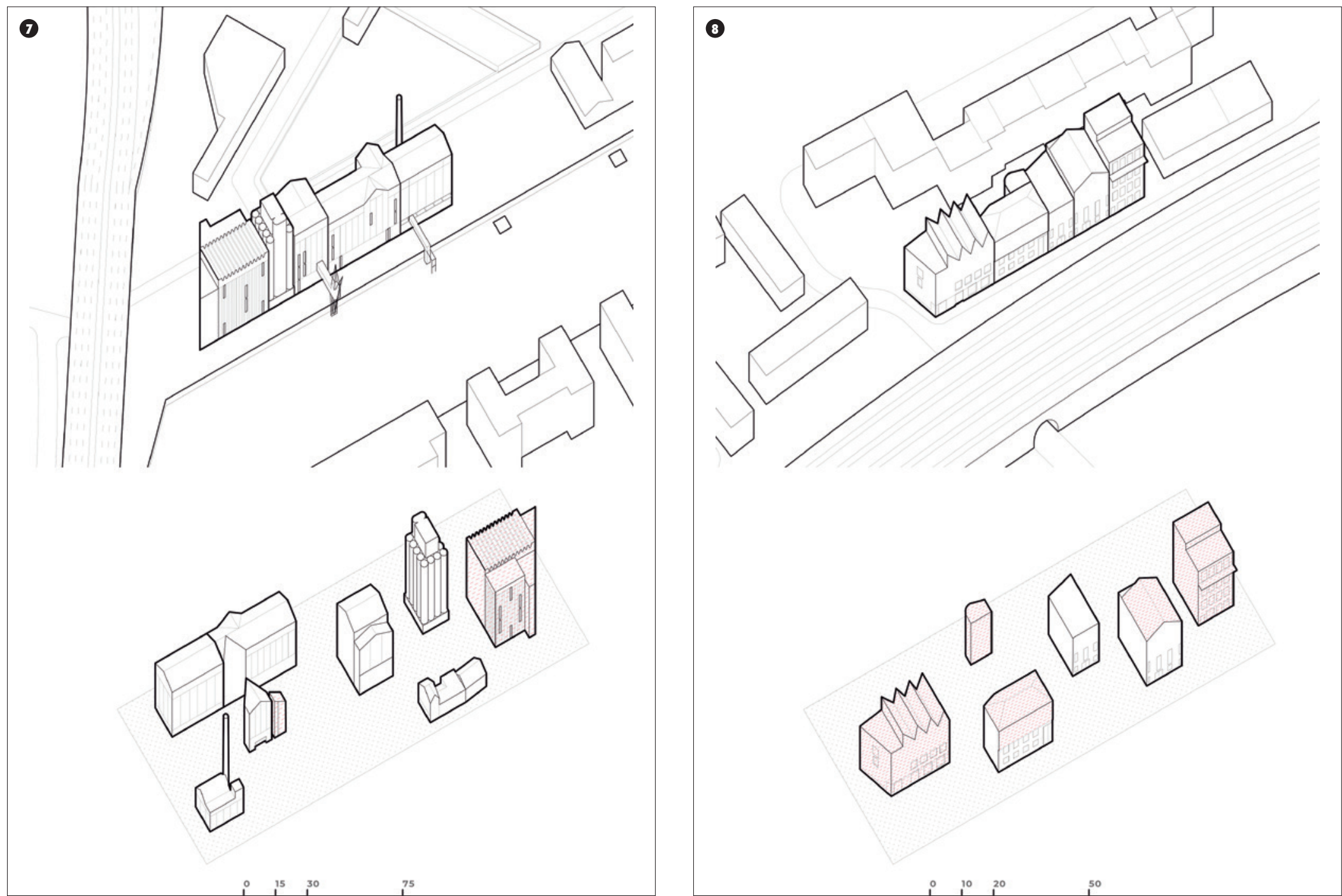

que habitualmente cuenta el museo. Se pretende lograr un lugar sin condiciones y sin interés por clasificar; una infraestructura de naturaleza urbana cuya razón de ser quede ligada a las plusvalías de la cultura contemporánea. Todo ello lo empareja con el primer Pompidou, compartiendo el objetivo de dar entrada en el escenario institucional a la cultura de masas y superar así lo que Andreas Huyssen ha denominado "gran división": el discurso que insiste en una distinción categórica entre la alta cultura y la cultura de masas (Huyssen, $2011)$. El primer Pompidou acometió este compromiso con la flexibilidad funcional, la neutralidad espacial y la apertura programática e institucional mediante el recurso de la transparencia (en deuda con la arquitectura moderna). Transparencia literal, materializada en el cerramiento de vidrio; planimétrica, al utilizar una superestructura que libera el espacio de pilares e impedimentos; e ideológica, al proponer una arquitectura del museo como lugar de la transparencia, la polivalencia, el consenso y el contrato social ${ }^{7}$. El nuevo Pompidou belga, sin renunciar a esta valiosa herencia, se muestra consciente del tiempo en el que nace, haciendo que la propia transparencia adquiera unas connotaciones diferentes. En este caso, se desprende de la apariencia

En su análisis del Pompidou, Jean Baudrillard señaló la polémica social implícita en la propuesta y la transformación del significado de cultura que suponía el centro parisino. Cabe añadir que el concepto de "producción" (por parte de los autores), también el de "infraestructura", remite a la noción promovida por Fred Jameson, que inspiró una renovada narración de los estadios de la cultura en relación con los modos de producción posindustrial (Jameson, 2015). 
9. (Izquierda) KANAL-Centre Pompidou, Bruselas, 2018-. Sergison Bates, EM2N, Noa. (Derecha) Gare Maritime, Bruselas, 2020. Neutelings Riedijk Architects. Fuente: www.sergisonbates.com_www.neutelingsriedijk.com / Fotógrafo: Filip Dujardin.
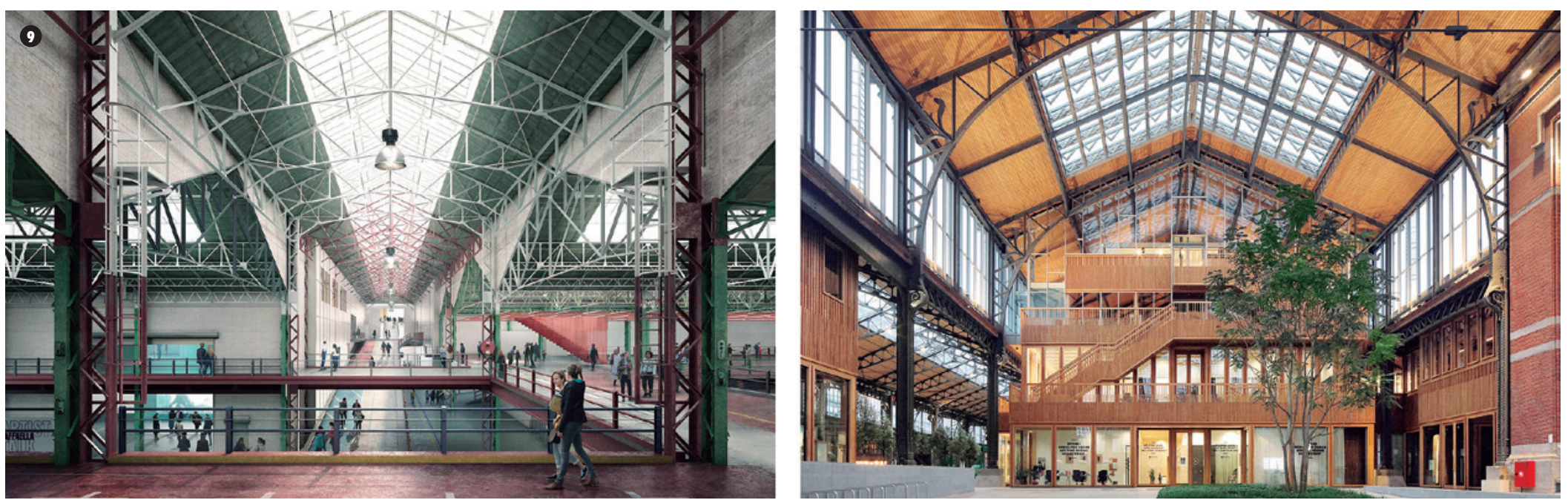

positivista y utópica para abrazar una actitud cercana al pragmatismo. Parece asumir, con ello, algunos de los planteamientos del arte contemporáneo, donde la visión ideal de una sociedad se ha sustituido por una visión donde el arte "simplemente" parece conformarse con alimentar una actitud crítica en el espectador y activar su propia energía creativa, como se aprecia en algunas obras de Gerhard Richter o Dan Graham (Boie y Rasker, 2018) (Figuras 9 y 10).

En la regeneración de este ámbito de Bruselas, también destaca el centro Gare Martime, basado en la reconversión de una estación de principios del siglo XX y que supone una valiosa aportación por parte de los arquitectos Neutelings \& Riedijk a la noción de infraestructura urbana $^{8}$. La propuesta se basa en la introducción de doce nuevos pabellones modulares de madera en las naves laterales para asumir los nuevos usos. La implementación de estos pabellones crea una organización que integra bulevares, calles, parques y plazas en un espacio interno y da una respuesta eficaz tanto al contexto urbano como a la estructura y corporeidad de la arquitectura previa. El espacio central queda disponible para eventos públicos, al que se añaden diversos jardines que sirven de transición entre éste y los pabellones laterales. Esta organización, a modo de boulevard urbano, acentúa la naturaleza de infraestructura urbana del conjunto y la idea de una "ciudad dentro de la ciudad" 9 .

\section{CONCLUSIONES}

Un aspecto esencial de los espacios de la cultura que hemos visitado es la introducción de cuestiones acerca de las posibilidades de reconversión del espacio de la modernidad. En todos ellos, la intervención establece un momento dialéctico de un nuevo límite y una

8 Cabe señalar que "sin las restricciones conservadoras que en otras arquitecturas inhiben la reconversión y con una abundancia de espacio disponible, son pocas y muy concretas las características que definen el espacio industrial que, logrando mudar de uso conservan buena parte de su corporeidad" (Ruescas, 16, X). Las intervenciones en KANAL y Gare Maritime logran una eficaz reconversión, una "reutilización adaptativa", desde la reconversión del carácter y la corporeidad de la arquitectura previa.

El número 278 de la revista A+ (2019) está dedicado a la regeneración de la capital belga concebida como "laboratorio para la futura ciudad europea" y donde destacan KANAL y Gare Maritime como motores del cambio (Visscher, 2019). 
10. KANAL-Centre Pompidou, Bruselas, 2018-. Sergison Bates, EM2N, Noa. Fuente: Dibujo de elaboración propia, 2021.

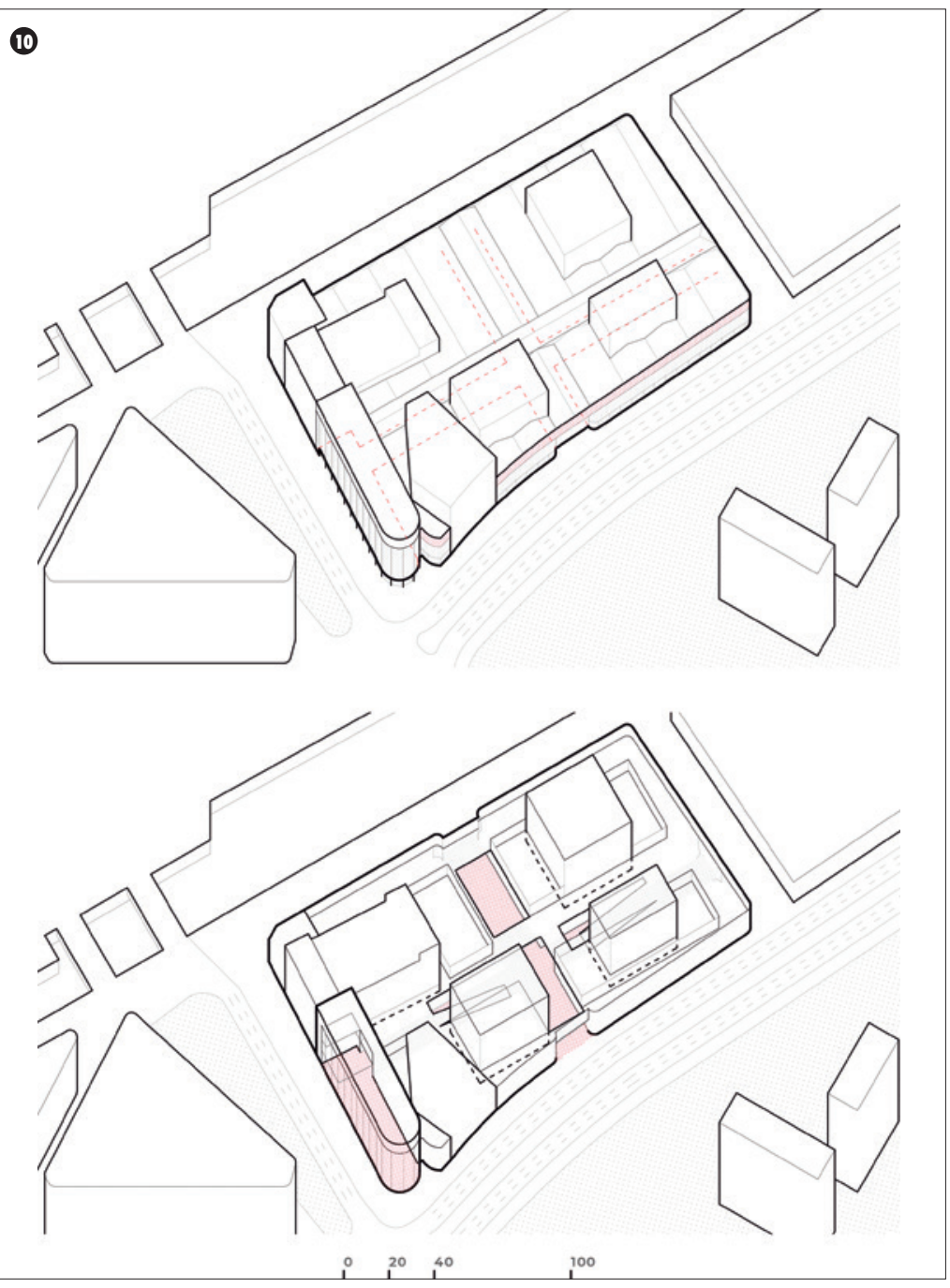

Cabe concluir señalando, que estos espacios culturales comparten que la producción artística de referencia ya no descansa en la disposición de unas obras privilegiadas, sino, más bien, en las distintas maneras de seleccionar o reciclar la sustancia cultural que es común a todos o, al menos, potencialmente accesible a todos. Ya apuntaba Paul Valèry en 1923 que las ideas de clasificación o conservación tenían poca relación con los deleites, y criticaba la confusión y descontextualización inherente al museo "¿̈He venido a instruirme, a buscar algo que me encante, o bien a cumplir con un deber y satisfacer las apariencias?" Junto a ello, se muestra plausible hacer frente a una lógica contemporánea híbrida que refuerce el intercambio entre arquitectura, arte y observador. En este sentido, lo que asegura la relevancia del museo o de los espacios de la cultura y el arte es su capacidad de adaptación a estos cambios, no la habilidad para aferrarse a una forma y contenidos establecidos de una vez para siempre.

\section{REFERENCIAS BIBLIOGRÁFICAS}

Abrahams, T. (2015). "Artists' gild". The Architectural Review, 238 (1423), 94-103.

Badía, J. (2012). "Nuevos museos en viejos edificios". Hertiage \& Museography 9, 4 (1), 20-25

Bauman, Z. (2011). La cultura en el mundo de la modernidad líquida. Madrid: FCE. Beck, U. (1998). La sociedad del riesgo: hacia una nueva modernidad. Barcelona: Paidós.

Bourriaud, N (2008). Estética relacional. Buenos Aires: Adriana Hidalgo.

Boie, G. \& Rasker, T. (2018). Please don't touch the museum: the urban art of the museum interior in the design competition for KANAL in Brussels. OASE, (101) 90-103.

Foster, H. (201 1). Art-Architecture complex. New York: Verso.

Foster, H. (2001). El retorno de lo real. Madrid: Akal.

Foster, H. (2010). New monumentality: Architecture and public space. Perspecta, (42), 135-139.

Hays, K. M. (2016). Architecture's appearance and the practices of imagination. Log, (37), 205-213

Hernández Martínez, A. 2013. De Museos, antimuseos y otros espacios expositivos en la Europa del siglo XXI. Artigrama, (28), 29-54.

Herzog, J.; De Meuron, P. (2018). Ampliación del Museo Küppersmühle, Duisburg. AV Proyectos, (89), 12-15.

Huyssen, A. (2010). Modernismo después de la modernidad. Barcelona: Gedisa. Jameson, F. (2015). "La estética de la singularidad". New Left Review, (92), 109 141.

Jameson, F. (1989). Posmodernism or The Cultural Logic of Late Capitalism. Durham: Duke UP.

Koolhaas, R.; Foster, H. (2013). Junkspace/Running room. London: Notting Hill Editions.

Lacaton, A.; Vassal, J. P. (201 1). "La libertad estructural, condición del milagro". 2G, (60), $161-175$.

Magni, C. (2018). "Fondazione Prada". Casabella, 82 (884), 80-99.

Mallgrave, H. F. (2018). From Object to Experience. London: Bloomsbury Visual Arts. Masoero, A. (2018). "Prada and the city". Domus, (1025), 116-125.

Mitchell, W.J.T. (2015). "Screening nature (and the nature of the screen)". New Review of Film and Television Studies, 13 (3), 231-246.

Moneo, R. (2019). Nuevos intereses, otros discursos. Pamplona: T6 ediciones.

Rorty, R. (1989). Contingency, Irony and Solidarity. New York: Cambridge UP.

Ruescas, J. (2016). Objetos tenaces. Adaptative Reuse en Meatpacking, 1970-1985. Tesis doctoral, ETSAM, Universidad Politécnica de Madrid.

Sloterdijk, P. (2020). El imperativo estético. Madrid: Akal.

Sloterdijk, P. (2015). Museum—School of Alienation. Art in Traslation, 6 (4), 437 448.

Visscher, L. (2019). Gare Maritime. A+, (278), 10-13.

Woodman, E. (2016). New and old are bound together. The Architectural Review, $240(1434), 16-21$ 